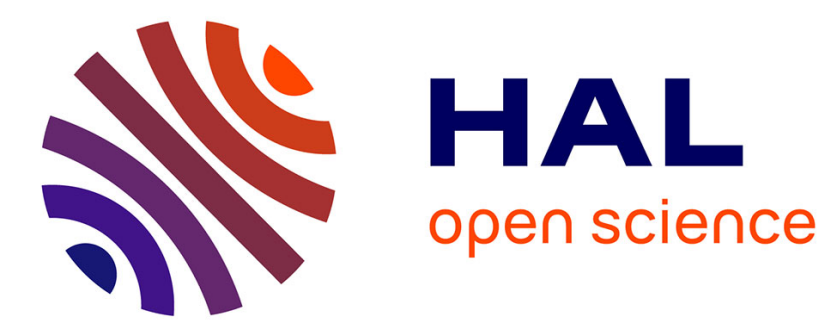

\title{
Local Anesthetics Inhibit the Growth of Human Hepatocellular Carcinoma Cells
}

G. Le Gac, G. Angenard, B. Clément, B. Laviolle, C. Coulouarn, H. Beloeil

\section{To cite this version:}

G. Le Gac, G. Angenard, B. Clément, B. Laviolle, C. Coulouarn, et al.. Local Anesthetics Inhibit the Growth of Human Hepatocellular Carcinoma Cells. Anesthesia \& Analgesia, 2017, 125 (5), pp.16001609. 10.1213/ANE.0000000000002429 . hal-01637191

\section{HAL Id: hal-01637191 \\ https://hal-univ-rennes1.archives-ouvertes.fr/hal-01637191}

Submitted on 28 Nov 2017

HAL is a multi-disciplinary open access archive for the deposit and dissemination of scientific research documents, whether they are published or not. The documents may come from teaching and research institutions in France or abroad, or from public or private research centers.
L'archive ouverte pluridisciplinaire HAL, est destinée au dépôt et à la diffusion de documents scientifiques de niveau recherche, publiés ou non, émanant des établissements d'enseignement et de recherche français ou étrangers, des laboratoires publics ou privés. 


\title{
Local Anesthetics Inhibit the Growth of Human Hepatocellular Carcinoma Cells
}

\author{
Grégoire Le Gac, MD,*† Gaëlle Angenard, BS,* Bruno Clément, PhD,* Bruno Laviolle, MD, PhD, $\neq$ \\ Cédric Coulouarn, $\mathrm{PhD}, *$ and Hélène Beloeil, MD, PhD*†
}

\begin{abstract}
BACKGROUND: Hepatocellular carcinoma (HCC) is an aggressive cancer with limited therapeutic options. Retrospective studies have shown that the administration of local anesthetics (LAs) during cancer surgery could reduce cancer recurrence. Besides, experimental studies reported that LAs could inhibit the growth of cancer cells. Thus, the purpose of this study was to investigate the effects of LAs on human HCC cells.

METHODS: The effects of 2 LAs (lidocaine and ropivacaine) $\left(10^{-2}\right.$ to $\left.10^{-6} \mathrm{M}\right)$ were studied after an incubation of 48 hours on $2 \mathrm{HCC}$ cell lines, namely HuH7 and HepaRG. Cell viability, cell cycle analysis, and apoptosis and senescence tests were performed together with unsupervised genome-wide expression profiling and quantitative real-time polymerase chain reaction for relevant genes.

RESULTS: We showed that LAs decreased viability and proliferation of HuH7 cells (from $92 \%$ $[P<.001]$ at $5 \times 10^{-3} \mathrm{M}$ to $40 \%[P=.02]$ at $10^{-4} \mathrm{M}$ with ropivacaine and from $87 \%$ $[P<.001]$ to $37 \%[P=.02]$ with lidocaine) and HepaRG progenitor cells (from $58 \%$ at $5 \times 10^{-3}$ $\mathrm{M}[P<.001]$ to $29 \%$ at $10^{-4} \mathrm{M}[P=.04]$ with lidocaine and $59 \%[P<.001]$ with ropivacaine $\left.5 \times 10^{-3} \mathrm{M}\right)$ in concentration-dependent manner. LAs have no effect on well-differentiated HepaRG. Ropivacaine decreased the mRNA level of key cell cycle regulators, namely cyclin A2, cyclin B1, cyclin B2, and cyclin-dependent kinase 1, and the expression of the nuclear marker of cell proliferation MKI67. Lidocaine had no specific effect on cell cycle but increased by $10 \times$ the mRNA level of adenomatous polyposis coli $(P<.01)$, which acts as an antagonist of the Wnt $/ \beta$ catenin pathway. Both LAs increased apoptosis in Huh7 and HepaRG progenitor cells $(P<.01)$. CONCLUSIONS: The data demonstrate that LAs induced profound modifications in gene expression profiles of tumor cells, including modulations in the expression of cell cycle-related genes that result in a cytostatic effect and induction of apoptosis. (Anesth Analg 2017;XXX:00-00)
\end{abstract}

Surgical tumor resection is a main treatment of solid cancers. However, surgery itself is associated with an increased risk of tumor cell dissemination and recur-

rence. ${ }^{1}$ Several reports suggested that the type of anesthesia chosen for surgery could be crucial and may influence the fate of the disease. Thus, a benefit of local anesthetic (LA) administration during cancer surgery has been suggested in several solid tumors (eg, in prostate cancer). ${ }^{2}$ LAs have long been used for their capacity to block nociceptive input. They are routinely used during and after surgery for their analgesics and anti-inflammatory properties. Perineural, perimedullar, or intravenous (only for lidocaine) administration of LAs were reported to improve postoperative rehabilitation by shortening postoperative ileus, length of

From the *INSERM, UMR 991, and Universite de Rennes 1, Rennes, France †CHU Rennes, Pôle Anesthésie et Réanimation, Inserm CIC 1414, Rennes, France; and $\ddagger$ CHU Rennes, Clinical Pharmacology Department and Inserm CIC 1414, Université de Rennes 1, Rennes, France.

Accepted for publication July 25, 2017

Funding: This research was supported by INSERM, University of Rennes 1, Ligue Contre le Cancer InCa/Canceropole and SFAR (French society of Anesthesia and Intensive Care) France.

The authors declare no conflicts of interest.

Supplemental digital content is available for this article. Direct URL citations appear in the printed text and are provided in the HTML and PDF versions of this article on the journal's website (www.anesthesia-analgesia.org).

Address correspondence to Hélène Beloeil, MD, PhD, Pôle d'Anesthésie Réanimation Chirurgicale, CHU Rennes, 2 Rue Henri Le Guilloux, 35033 Rennes Cedex 9, France. Address e-mail to helene.beloeil@chu-rennes.fr. stay, and improving analgesia. ${ }^{3}$ Retrospective studies have suggested that the administration of LAs during cancer surgery could reduce cancer recurrence. ${ }^{2,4,5}$ A meta-analysis of 14 studies showed a better overall survival when general anesthesia was associated with an epidural analgesia during cancer surgery. ${ }^{6}$ Large-scale prospective clinical studies are currently recruiting to further investigate this potential benefit of LAs. Besides, experimental studies have reported an inhibitory effect of LAs on tumor cell growth in lung and colon cancer. ${ }^{7,8}$ LAs were notably reported to activate caspases and to decrease estimated glomerular filtration rate activity. These effects vary with the type and the concentration of LAs used, and the type of cancer. ${ }^{7}$ While studies in several solid tumors (eg, lung) have been published, very few reports were related to hepatocellular carcinoma (HCC) so far. Interestingly, one of these studies reported that procaine exhibits growth inhibitory and DNA demethylating effects on HCC cells. ${ }^{9}$ Notably, procaine was shown to restore the expression of tumor suppressor gene CDKN2A/ $\mathrm{P} 16$, which is frequently silenced by promoter hypermethylation in cancer. ${ }^{9}$ However, procaine is rarely used in clinical medicine. HCC is a frequent and aggressive cancer with limited therapeutic options. According to the Barcelona Centre Liver Cancer classification, ${ }^{10}$ surgery is recommended for early-stage HCC (Barcelona Centre Liver Cancer classification 0 ). In this context, patients are stringently selected, and surgery is restricted to patients with solitary tumors and very well-preserved liver function, as normal bilirubin with either hepatic venous pressure gradient $\leq 10 \mathrm{~mm} \mathrm{Hg}$ or platelet count $\geq 100,000 .{ }^{10}$ However, the benefit of surgery is limited by a high risk of tumor recurrence $(70 \%$ after 
5 years). ${ }^{11}$ In the present study, we investigated the effects of 2 LAs (ropivacaine and lidocaine) on the growth of $\mathrm{HuH}^{12}$ and HepaRG human HCC cells. HepaRG cell line exhibits a unique property to differentiate into well-differentiated hepatocytes from highly proliferative progenitor cells. ${ }^{13}$ We have previously shown that HepaRG cells represent a suitable model in HCC carcinogenesis. ${ }^{14,15}$ We hypothesized that LAs would specifically inhibit the viability and proliferation of HCC cells.

\section{METHODS}

\section{Cell Lines and Experimental Procedure}

$\mathrm{HuH7}$ and HepaRG cell lines were established and maintained as previously described. ${ }^{12,16} \mathrm{HuH7}$ cells were grown in Dulbecco's Modified Eagle's medium (with L-glutamine-D glucose without pyruvate sodium) supplemented with $10 \%$ fetal bovine serum, $1 \%$ penicillin/streptomycin. HepaRG cells were grown in William's E medium supplemented with $10 \%$ fetal bovine serum, $1 \%$ penicillin/streptomycin, $5 \mu \mathrm{g} / \mathrm{mL}$ insulin, and $50 \mu \mathrm{mol} / \mathrm{L}$ hydrocortisone hemisuccinate. Differentiation of HepaRG cells from proliferative progenitors to mature well-differentiated hepatocytes was achieved in 4 weeks by culturing the cells in the supplemented medium in the presence of $2 \%$ dimethyl sulfoxide for the last 2 weeks as previously described. ${ }^{14}$ All cell cultures were conducted at $37^{\circ} \mathrm{C}$ in a $5 \% \mathrm{CO}_{2}$ atmosphere. Independent culture experiments were performed at least in triplicate. It was not feasible for the experimenters (G.L.G., H.B., and G.A.) to be blind to the experimental conditions.

Cells were incubated with or without LAs for 24, 48, or 72 hours. Ropivacaine $(7.5 \mathrm{mg} / \mathrm{mL})$ (Kabi, Heudebouville, France) and lidocaine (10 mg/mL) (Aguettant, Lyon, France) were diluted with the corresponding cell culture medium depending on the cell line (see above) to achieve the tested concentrations (from $10^{-2}$ to $10^{-5} \mathrm{M}$ ). Concentrations of LAs were fixed over time (for 24 or 48 hours) except for 1 set of experiments in which the effects of decreasing LA concentrations $\left(10^{-3} \mathrm{M}\right.$ [day 1 ], $10^{-4} \mathrm{M}$ [day 2], $10^{-5} \mathrm{M}$ [day 3]) were assessed over 72 hours. Concentrations, number of cells, and number of experiments were chosen in accordance with previous published data., ${ }^{74}$

\section{Cell Viability and Proliferation}

Cells were seeded into 96-well plates (20,000 cells per well for $\mathrm{HuH7}$ and HepaRG progenitors and 50,000 cells per well for differentiated HepaRG cells). Viability was assessed at 24,48 , or 72 hours with a MTT colorimetric assay. ${ }^{17}$ Cytotoxicity of ropivacaine and lidocaine on Huh7 and HepaRG progenitors was evaluated by measuring lactae deshydrogenase (LDH) activity (Pierce LDH cytotoxicity assay; Thermofisher). LDH assay determines the release of cytoplasmic enzyme lactate dehydrogenase due to plasma membrane damage. LDH assay results were expressed in percentage of cytotoxicity by subtracting the LDH activity of the spontaneous LDH release control (water treated) from the chemical-treated sample LDH activity, divided by the total LDH activity [(maximum LDH release control activity) - (spontaneous LDH release control activity)], and multiplied by 100, following manufacturer instructions. DNA synthesis was assessed using the Click-iT Plus Edu
Assay (Molecular Probes). Edu assay results were expressed in percentage of marked cell, by dividing the number of stained cell by the number of nucleus assessed by Hoechst.

\section{Cell Cycle Analysis}

Cell cycle phases were analyzed using a Cellomics Arrayscan Vti (Thermoscientific Laboratory, Villebon-sur-Yvette, France). Cells were seeded into 96-well plates with or without LAs. After 48 hours, cells were fixed for 20 minutes at $4^{\circ} \mathrm{C}$ with an acetic alcohol solution. Fixative was removed and each well was washed with PBS. Cells were then incubated with a Hoechst solution for 20 minutes, washed, and dried. Images were captured using an Olympus microscope and Cellomics Arrayscan Vti, allowing cell quantification in different phases of the cell cycle. Experiments were performed on HuH7 and HepaRG progenitors cells treated with $10^{-4} \mathrm{M}$ lidocaine and ropivacaine.

\section{Apoptosis Test}

The activity of caspase-3/7 (CPP32/apopain)-like proteases was determined using the EnzChek Caspase-3 Assay Kit following manufacturer instructions. Briefly, $5 \times 10^{5} \mathrm{HepaRG}$ cells on 6-well plates were incubated with or without LAs following the same procedures as the MTT assay. After 48 hours, the cells were washed with PBS, lysed, and caspase activity in the extracts was measured. Fluorescent product of the Z-DEVDrhodamine 110 substrate generated by caspase-3-like proteases was detected by a Polarstar Omega fluorometer with excitation/emission at 496/520 nm. Background fluorescence was determined by following the same procedures without cells and subtracted from the total. Negative control was performed by including a specific caspase-3 inhibitor (Ac-DEVD-CHO) in HepaRG cells. Positive control was performed by treating HepaRG cells with doxorubicin $(50 \mathrm{ng} / \mathrm{mL}) .{ }^{18}$ Apoptosis was also detected in situ by using a Click-iT Plus TUNEL assay (Molecular Probes, Villebon-sur-Yvette, France). Results were expressed in percentage of marked cell by dividing the number of stained cells by the number of nuclei assessed by Hoechst.

\section{Senescence Test}

Senescence-associated $\beta$-galactosidase activity was detected with a Cellular Senescence Assay Kits KAA002 (Merck Millipore, Saint Quentin-en-Yvelines, France). Cells were grown with LAs, washed in PBS, fixed for 3-5 minutes at room temperature in $2 \%$ formaldehyde $/ 0.2 \%$ glutaraldehyde, washed, and incubated at $37^{\circ} \mathrm{C}$ (No. C02) with fresh senescence-associated (3-Gal [SA-, 3-Gal]) stain solution (1 $\mathrm{mg}$ of 5 bromo-4-chloro-3-indolyl P3-D-galactoside [X Gal] per $\mathrm{mL} / 40 \mathrm{mM}$ citric acid/sodium phosphate, $\mathrm{pH} 6.0 / 5$ $\mathrm{mM}$ potassium ferrocyanide $/ 5 \mathrm{mM}$ potassium ferricyanide/150 $\mathrm{mM} \mathrm{NaCl} / 2 \mathrm{mM} \mathrm{MgCl}$ ). Then stained cells were count under optical microscopy.

\section{Microarray Analysis}

Total RNA was purified from cells with miRNeasy Mini Kit (Qiagen, Courtaboeuf, France). Quantity and quality of RNA were evaluated with a Nanodrop ND-1000 spectrophotometer (Nyxor, Palaiseau, France). Genome-wide expression profiling was conducted using a 1-color, low-input Quick Amp Labelling Kit and human SurePrint G3 8x60K pangenomic microarrays (Agilent Technologies, Les Ulis, France), 
as previously described. ${ }^{19}$ Briefly, differentially expressed genes were identified by a 2 -sample univariate $t$ test with a random variance model. Individual genes were selected on the basis of both statistical significance $(P<.01)$ and fold change (FC) difference between the compared groups (FC $>1.5$ ). Microarray experiments were performed on HuH7 cells treated with $10^{-3} \mathrm{M}$ lidocaine and ropivacaine for 48 hours. Microarray data mining was performed as previously described using Gene Set Enrichment Analysis and Gene Ontology data mining tools. ${ }^{19}$

\section{Real-Time Reverse Transcriptase Polymerase Chain Reaction}

Relevant genes from microarray data were chosen to confirm the effects of LAs $\left(10^{-3} \mathrm{M}\right.$ and $\left.10^{-4} \mathrm{M}\right)$ on HuH7 and HepaRG cells. Gene expression was measured by quantitative real-time polymerase chain reaction (QRT-PCR), as done previously. ${ }^{14}$ Quantitative analysis of PCR data was conducted with the $2^{-\Delta \Delta C t}$ method using $\beta$-actin $C t$ values for normalization. Melting analysis was conducted to validate the specificity of PCR products. The list of oligonucleotides used for QRT-PCR experiments is provided in Supplemental Digital Content 1, Table 1, http://links.lww. com/AA/B968.

\section{Western Blot}

Protein extraction was performed after 48 hours of LA treatment using a RIPA Lysis and Extraction Buffer (Life Technologies, Marly-le-Roi, France). Protein concentration was determined with a Pierce bovine serum albumine (BSA) Protein Assay kit (Thermo Scientific) by absorbance measurement at $562 \mathrm{~nm}$. NuPAGE LDS Sample Buffer (4X) and NuPAGE Sample Reducing Agent (10X) (Life Technologies) were mixed with $30 \mu \mathrm{g}$ of protein; $\mathrm{H}_{2} \mathrm{O}$ was added for a final volume of $15 \mu \mathrm{L}$. The mixture was incubated for 10 minutes at $70^{\circ} \mathrm{C}$. After gel migration for 45 minutes at 200 V (NuPAGE Novex $4 \%-12 \%$ and 20X NuPAGE MOPS SDS Running Buffer, Life Technologies), proteins were transferred into a membrane using a Blot Dry Blotting System (Invitrogen, Paris, France). Nonspecific sites were saturated with an ECL Advance Blocking Agent (GE Healthcare, Velizy, France). Primary and secondary antibodies were diluted $(1 / 10,000)$ in BSA $3 \%$ and tris buffered saline (TBS) $1 \%$. Antibodies were incubated for at least 1 hour (APC Antibody [C-20], sc-896 Santa Cruz Biotechnology; antirabbit Antibody, DAKKO; Cyclin antibody sampler Kit \#9869; Cell Signal Technology, Saint Quentin-en-Yvelines, France). The detection was performed with an ECLAdvance Western Blotting Detection Kit (GE Healthcare). Quantification was achieved by densitometry analysis.

\section{Statistical Analysis}

Normal distribution of data was assessed with a ShapiroWilk test. The effects of the different concentrations of LAs on HCC viability were compared using a 2-way (cell type, LAs concentration) analysis of variance. In case of significant concentration or cell effect or of cell $\times$ concentration interaction (when comparing the effects of the different concentrations of LAs on HCC viability), pair-wise comparisons were performed using the Tukey test to control the overall rate of type I error due to multiple comparisons. Other comparisons between quantitative variables were performed using the Student $t$ test or Wilcoxon rank sum test when needed. In these analyses, $P<.05$ after adjustment for multiple comparisons was considered statistically significant. Results are presented as percentages of variation between the mean of the group of interest versus control. In the microarray analysis, we identified genes that were differentially expressed among the 2 classes using a random-variance $t$ test. The random-variance $t$ test is an improvement over the standard separate $t$ test as it permits sharing information among genes about within-class variation without assuming that all genes have the same variance. ${ }^{20}$ The genes were considered to be differentially expressed between the 2 conditions (treated versus control) when $P<.01$ and a FC $>1.5$. A more stringent statistical threshold was also applied for ropivacaine versus control: $P<.001$ and FC $>2$. For experiment on cell viability (Figure 1), a sample size of at least 7 experiments per group allowed to have $97 \%$ power to detect at the 0.050 level a difference in means characterized by a variance of means of 0.042 (corresponding to expected values of 1.3 in $\mathrm{HuH7}$ group, 1 in HeparRG, and 0.8 in HepaRG progenitors cells), assuming that the common standard deviation is 0.200 . These differences corresponded to an expected benefit of at least $25 \%$ with ropivacaine and $60 \%$ with lidocaine as compared with placebo. With our results, 6 experiments per group for lidocaine and 8 per group for ropivacaine were sufficient to detect the observed difference with $90 \%$ power. For experiments on mRNAs levels of genes of interest (Figure 3), QRTPCR (Figures 4 and 5), and caspase activity, a sample size of at least 3 experiments per group allowed to have $90 \%$ power to detect an effect size of at least 3.6 for ropivacaine and lidocaine at the 0.050 level using a 2-group $t$ test. The statistical analysis was performed using SAS statistical software V9.3 (SAS Institute, Cary, NC).

\section{RESULTS}

\section{Lidocaine and Ropivacaine Reduce Cell Viability of Proliferative Tumor Cells}

The effects of LAs on cell viability were first evaluated on $\mathrm{HuH7}$ and HepaRG cell lines at 48 hours (Figure 1). There was a significant cell $\times$ concentration interaction $(P<.001)$. Pair-wise comparisons showed that as compared with control, lidocaine and ropivacaine (concentrations ranging from $5 \times 10^{-3} \mathrm{M}$ to $10^{-4} \mathrm{M}$ ) significantly decreased the growth of HuH7 cells as follows: by $87 \%(P<.001)$ and $92 \%(P<.001)$ at $5 \times 10^{-3} \mathrm{M}, 35 \%(P=.02)$ and $67 \%(P<.001)$ at $10^{-3} \mathrm{M}, 37 \%$ $(P=.02)$ and $40 \%(P=.02)$ at $10^{-4} \mathrm{M}$, respectively. Lidocaine and ropivacaine also decreased the proliferation of highly proliferative HepaRG progenitors (at concentrations of $5 \times 10^{-3} \mathrm{M}[58 \% ; P<.001], 10^{-3} \mathrm{M}[35 \% ; P<.01]$, and $10^{-4} \mathrm{M}$ $[29 \% ; P=.04]$ for lidocaine, and only at concentration of 5 $\times 10^{-3} \mathrm{M}[59 \% ; P<.001]$ for ropivacaine) but did not affect viability of hepatocyte-like differentiated HepaRG cells. Lidocaine and ropivacaine at $10^{-2} \mathrm{M}$ induced an important cytotoxicity on HuH7 cells (Supplemental Digital Content 2, Figure 1, http:/ /links.lww.com/AA/B969) and therefore this concentration was not further used in the experiments. No effect on cell viability and proliferation of the cell lines were observed with the lowest concentrations $\left(10^{-5}\right.$ and $\left.10^{-6} \mathrm{M}\right)$. Cell damage assessed with LDH release test confirmed that only the highest concentrations were cytotoxic 


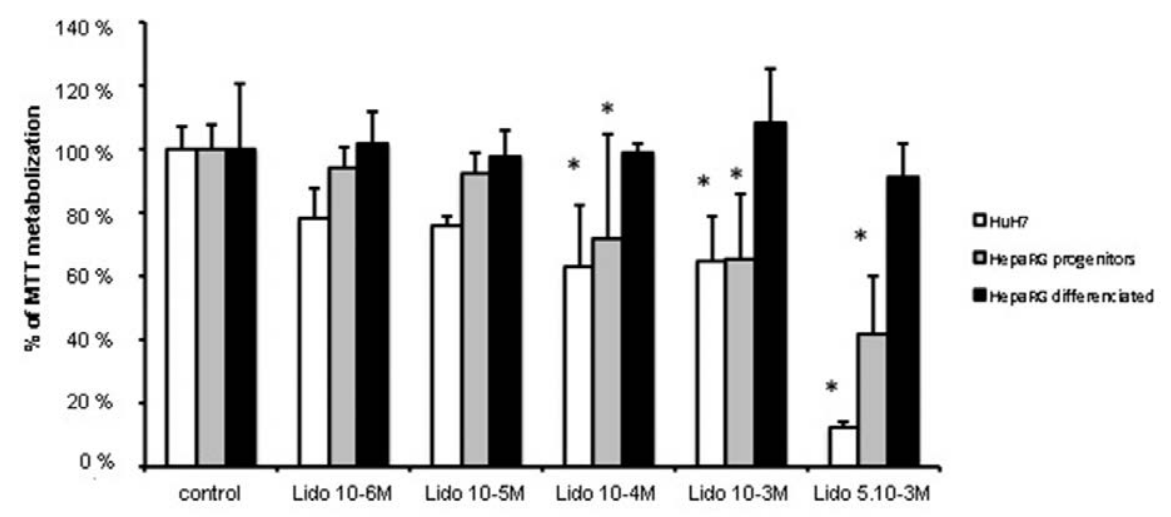

Figure 1. Effects of LAs on HCC cell growth. Lidocaine and ropivacaine significantly inhibited the growth of $\mathrm{HuH} 7$ cells and highly proliferative HepaRG progenitors but not of hepatocyte-like differentiated HepaRG cells. $\mathrm{HuH} 7$, HepaRG progenitors, and differentiated HepaRG cell lines were cultured in the presence of indicated concentrations of lidocaine (A) and ropivacaine (B) for $48 \mathrm{~h}$ and cell viability was assessed by MTT assay. In all graphs, data are mean \pm SD of more than 3 independent experiments $\left(n=7\right.$ for the $10^{-5}$ and $10^{-6} \mathrm{M}$ concentrations and $\mathrm{n}=9$ for all the other experiments). ${ }^{*} P<.05$ versus control. HCC indicates hepatocellular carcinoma; LA, local anesthetics; Lido, lidocaine; M, molar; Ropi, ropivacaine.

on HuH7 and HepaRG progenitors cell lines (Supplemental Digital Content 3, Table 2A, http://links.lww.com/AA/ B970). Inhibition of cell proliferation was supported by a LAs-dependent inhibition of DNA synthesis (Supplemental Digital Content 3, Table 2B, http://links.lww.com/AA/ B970). After 24 hours, the effect was less pronounced: The only significant effect was observed at $10^{-3} \mathrm{M}$ of lidocaine and ropivacaine, which significantly decreased the growth of HuH7 cells by $13 \%(P<.001)$ and $14 \%(P<.001)$, respectively. When testing the effects of decreasing concentrations of LAs over time, lidocaine and ropivacaine significantly decreased the growth of $\mathrm{HuH7}$ cells by $50 \%(P=.01)$ and $77 \%(P<.001)$ at 72 hours, respectively.

\section{Lidocaine Inhibits the Growth of HCC Cells by Increasing the Caspase 3 Activity}

The observed reduced cell viability induced by LAs on highly proliferative cells prompted us to determine whether LAs impact the cell cycle. Lidocaine had no significant effect on the cell cycle but was associated with an increase in the number of apoptotic bodies (Figure 2). Unsupervised genome-wide expression profiling showed in Huh7 cells that lidocaine treatments $\left(10^{-3} \mathrm{M}\right)$ for 48 hours resulted in the deregulation of 194 genes $(P<.01 ; \mathrm{FC}>1.5)$ (Supplemental Digital Content 4, Figure 2A, http://links.lww.com/AA/B971 and Supplemental Digital Content 3, Table 2, http:/ /links.lww.com/AA/B970). No drastic effect of lidocaine on mRNA levels of cell cycle regulators or cyclins was observed (Supplemental Digital Content4, Figure 2, http:/ /links.lww.com/AA/B971 and Supplemental Digital Content 5, Table 3, http:/ /links.lww.com/AA/B972). However, lidocaine increased by 10 times the mRNA levels of adenomatous polyposis coli $(A P C)(\mathrm{FC}>1.5 ; P<.01)$, which acts as an antagonist of the Wnt/ $\beta$-catenin pathway (Figure
3). Lidocaine had no effect on the expression of apoptosisrelated genes and protein such as caspases and poly (adenosin diphosphate (ADP) ribose) polymerases but increased mRNA levels of Harakiri (HRK), encoding a proapoptotic protein (Figure 3). QRT-PCR confirmed microarray results for genes coding for CCNA2 (no significant effect), CCNB1 (no significant effect), APC (on HuH7: 1800\% increase at 10-4 $\mathrm{M}[P=.01], 4400 \%$ increase at $10^{-3} \mathrm{M}[P<.01]$ and on HepaRG progenitors: $400 \%$ increase at $10^{-4} \mathrm{M}[P=.03], 300 \%$ increase at $10^{-3} \mathrm{M}[P=.04]$ ), and HRK (on HuH7: $48 \%$ increase at $10^{-4}$ $\mathrm{M}[P=.02], 129 \%$ increase at $10^{-3} \mathrm{M}[P<.01]$ and on HepaRG progenitors: $135 \%$ increase at $10^{-4} \mathrm{M}[P=.05], 117 \%$ increase at $10^{-3} \mathrm{M}[P<.01]$ ) (Figure 4). Lidocaine-induced gene deregulations were similar for both cell lines (Figure 4). Western-blot analysis showed that lidocaine increases APC protein level (on HuH7: $25 \%$ increase at $10^{-4} \mathrm{M}[P<.001], 32 \%$ increase at $10^{-3} \mathrm{M}[P<.001]$ and on HepaRG progenitors: $59 \%$ increase at $10^{-4} \mathrm{M}[P=.05], 54 \%$ increase at $10^{-3} \mathrm{M}[P=.02]$ ) but has no significant impact on the expression of cyclins (Supplemental Digital Content 6, Figure 3, http:/ /links.lww.com/AA/B973). Apoptosis was upregulated by lidocaine as demonstrated by caspase 3 activity (on HuH7: $393 \%$ increase at $10^{-4} \mathrm{M}[P<.01]$, $357 \%$ increase at $10^{-3} \mathrm{M}[P<.001]$ and on HepaRG progenitors: $104 \%$ increase at $10^{-4} \mathrm{M}[P<.01], 90 \%$ increase at $10^{-3} \mathrm{M}$ $[P<.01]$ ) (Figure 6) and TUNEL assays (Supplemental Digital Content 3, Table 2, http:/ /links.lww.com/AA/B970). No effect on senescence was observed (Supplemental Digital Content 7, Figure 4, http:/ /links.lww.com/AA/B974).

\section{Ropivacaine Inhibits the Growth of HCC Cells by Stopping the Cell Cycle in G2 Phase}

Cell cycle analysis demonstrated that ropivacaine treatment resulted in a drastic enrichment of cells in the G2 


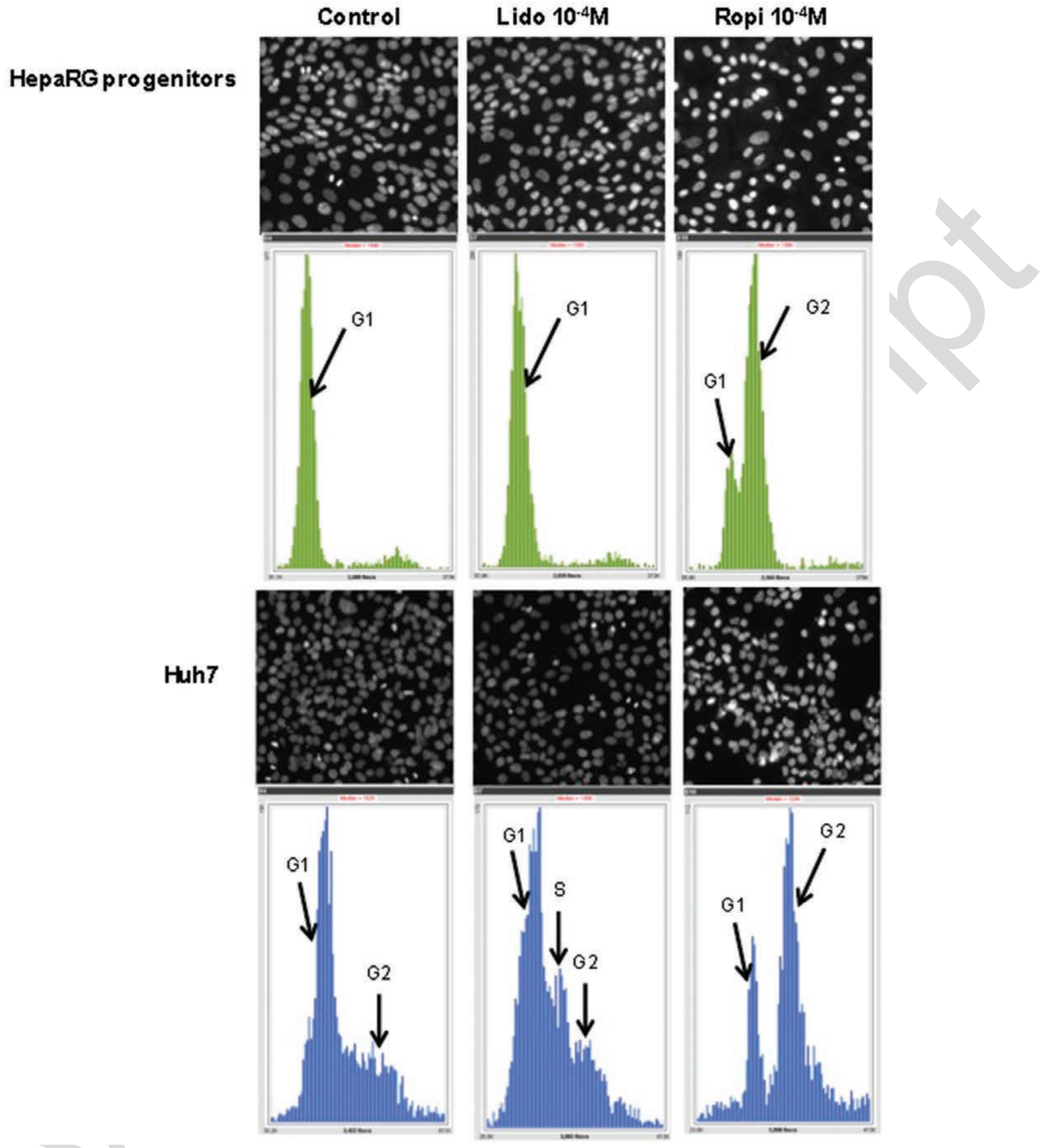

Figure 2. LAs induced cell cycle alterations. Cell cycle phases were analyzed using Cellomics Arrayscan Vti with or without (control) $10^{-4} \mathrm{M}$ lidocaine or ropivacaine after 48-h culture of $\mathrm{HuH} 7$ and HepaRG progenitors cells. Control: presence of mitotic nuclei and mitosis. Lido $10^{-4}$ $\mathrm{M}$ : reduction of mitotic nuclei and no significant effect on the cell cycle. Ropi $10^{-4} \mathrm{M}$ : absence of mitotic nuclei and accumulation of cells in the G2 phase for both cell lines. G1 indicates G1 phase; G2, G2 phase; LA, local anesthetics; M, molar; S, S phase.

phase for both cell lines, suggesting a cell cycle blockade before mitosis. This observation coincided with the absence of mitotic nuclei (Figure 2). Unsupervised genome-wide expression profiling in $\mathrm{HuH7}$ cells showed that ropivacaine treatment $\left(10^{-3} \mathrm{M}\right.$ for 48 hours) resulted in the deregulation of 221 genes $(P<.01 ; \mathrm{FC}>1.5)$ (Supplemental Digital Content 4, Figure 2B, http://links.lww.com/AA/B971 and Supplemental Digital Content 8, Table 4, http:/ /links. lww.com/AA/B975). Interestingly, ropivacaine was associated with a decrease in the expression of key cell cycle regulator genes, especially involved in the G2-M transition phase, namely cyclin A2 (CCNA2) (63\% decrease; $P<.01)$, cyclin B1 (CCNB1) (64\% decrease; $P=.02)$. Ropivacaine also decreased the expression of MKI67 a nuclear marker of cell proliferation $(61 \%$ decrease; $P<.01)$ (Figure 3$)$. Gene Set Enrichment Analysis confirmed the negative enrichment of cell cycle-associated gene signatures in $\mathrm{HuH7}$ cells treated with ropivacaine (Supplemental Digital Content 9, Figure 5, http://links.lww.com/AA/B976). QRT-PCR validated the microarray data for CCNA 2 (on HuH7: 81\% decrease at 
CCNA2

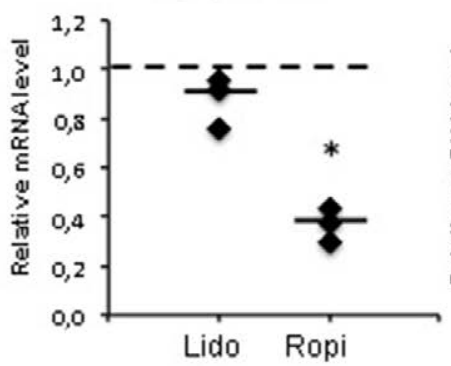

MKI67

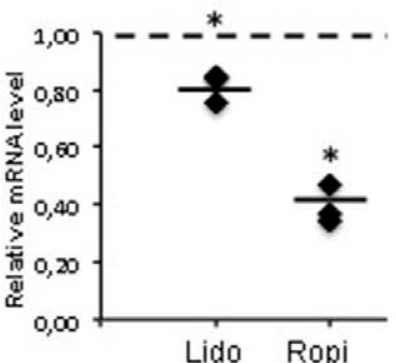

CCNB1

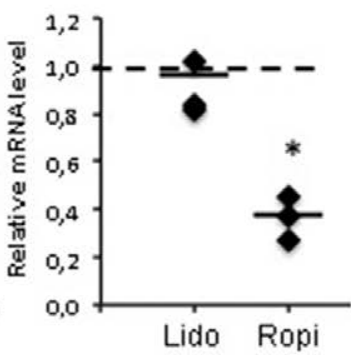

HRK

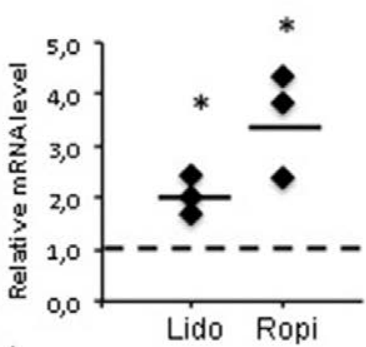

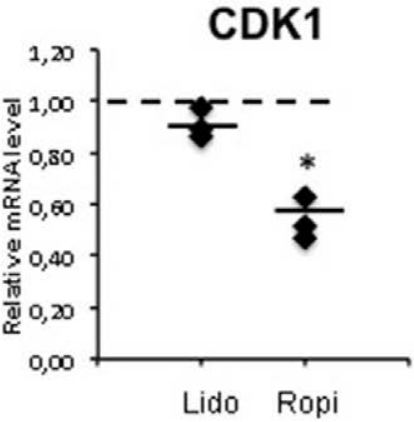

APC

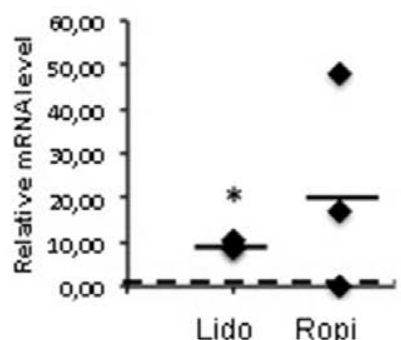

CCNBP1

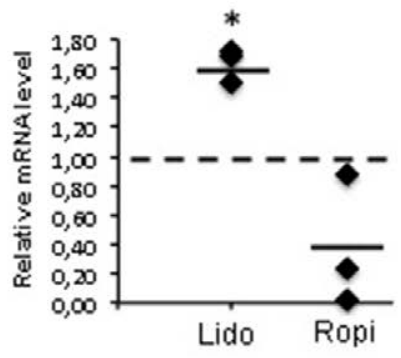

DKK1

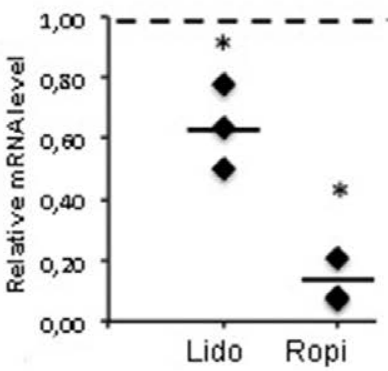

Figure 3. mRNAs levels of genes of interest detected by microarray after 48-h culture of HuH7 cells with or without $10^{-3} \mathrm{M}$ lidocaine and ropivacaine. Lidocaine significantly increased the mRNA levels of CCNDBP1 and APC. Besides MKI67, a nuclear marker of cell proliferation, lidocaine has no impact on the expression of cell cycle-associated genes. Ropivacaine decreased the mRNA level of key cell cycle regulators: CCNA2, CCNB1, CCNB2, and CDK1 and of MKI67. Both LAs increased the mRNA levels of HRK, apro-apoptotic protein. Data from 3 independent experiments shown as scatter plot (dashed line $=1$, representing control). Horizontal line indicates mean for each group. $* P<.05$ versus control. APC indicates adenomatous polyposis coli; CCNA2, cyclin A2; CCNB1, cyclin B1; CCNDBP1, cyclin D binding protein 1; HRK, Harakiri.

A $\quad$ CCNA2

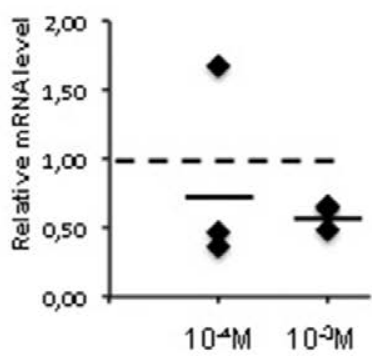

B

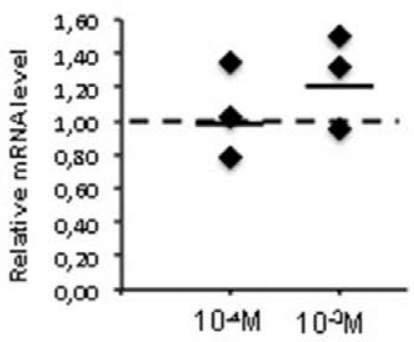

CCNB1

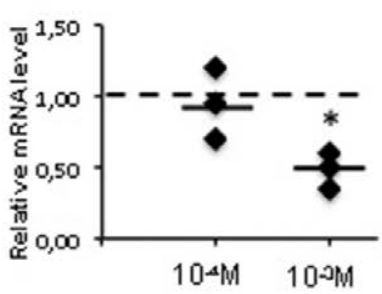

CCNB1
APC

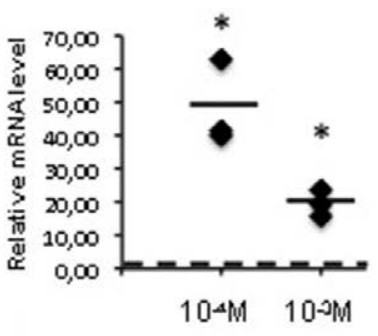

APC
HRK

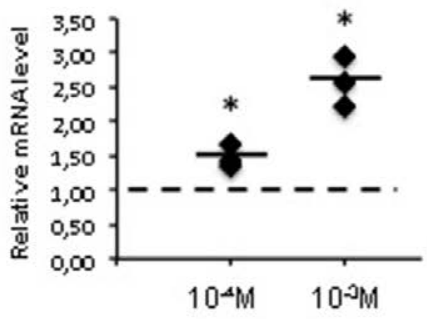

B CCNA2

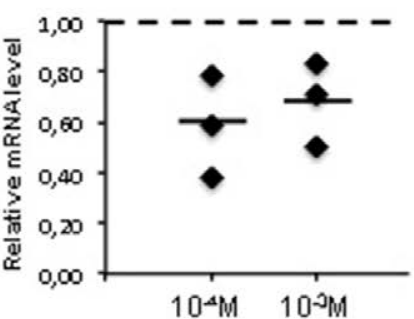

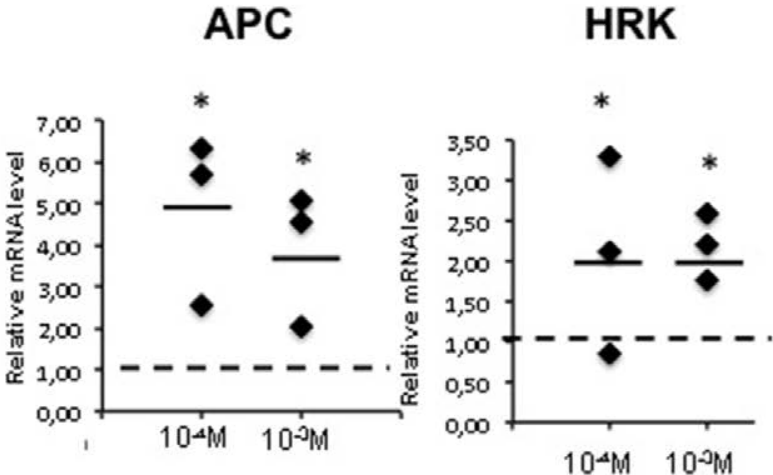

Figure 4. Quantitative real-time polymerase chain reaction confirmed the microarray data on HuH7 (A) and HepaRG progenitor (B) cells treated with lidocaine. Lidocaine induced a significant upregulation of APC and HRK. Lidocaine-induced gene deregulations were similar for both cell lines. Data from 3 independent experiments shown as scatter plot (dashed line $=1$, representing control). Horizontal line indicates mean for each group. ${ }^{*}<$ < .05 versus control. APC indicates adenomatous polyposis coli; CCNA2, cyclin A2; CCNB1, cyclin B1; HRK, Harakiri. 
A

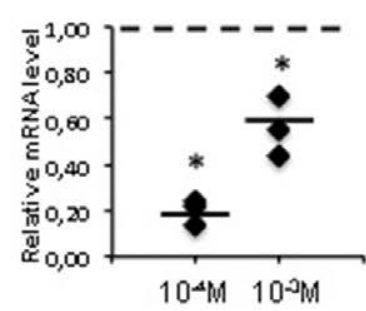

B

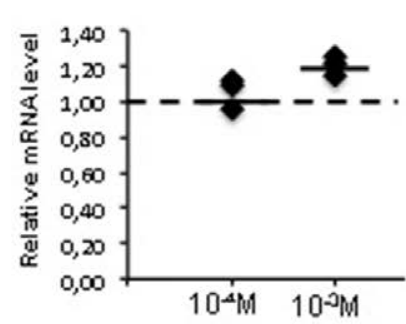

CCNB1

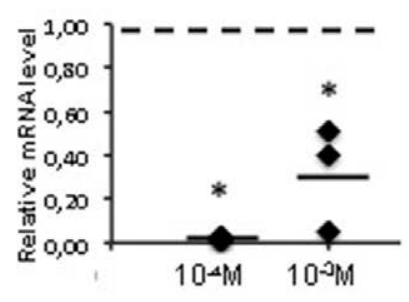

CCNB1

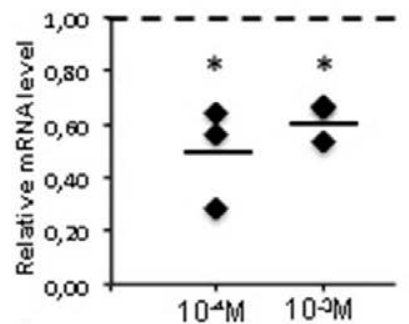

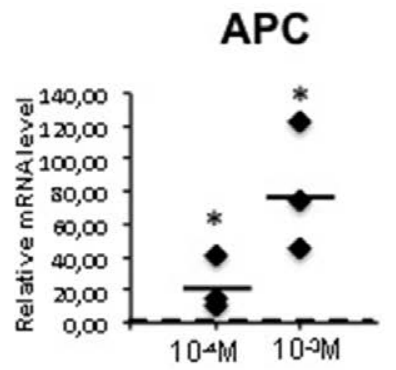

APC

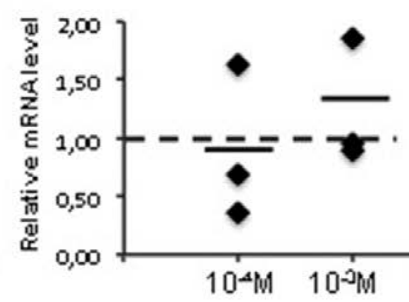

HRK

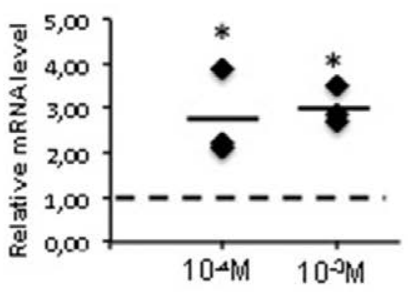

HRK

Figure 5. Quantitative real-time polymerase chain reaction confirmed the microarray data on $\mathrm{HuH}$ ( $\mathrm{A}$ ) and HepaRG progenitor (B) cells treated with or without ropivacaine. Ropivacaine significantly down-regulated CCNA2 and CCNB1 and upregulated HRK. Ropivacaine-induced gene deregulations were more pronounced in HuH7 cells. Data from 3 independent experiments shown as scatter plot (dashed line $=1$, representing control). Horizontal line indicates mean for each group. ${ }^{*} P<.05$ versus control. APC indicates adenomatous polyposis coli; CCNA2, cyclin A2; CCNB1, cyclin B1; CCNB2, cyclin B2; HRK, Harakiri; M, molar.

$10^{-4} \mathrm{M}[P<.01], 44 \%$ decrease at $10^{-3} \mathrm{M}[P=.01]$ and on HepaRG progenitors: not significant), CCNB1 (on HuH7: $98 \%$ decrease at $10^{-4} \mathrm{M}[\mathrm{P}=.01], 68 \%$ decrease at $10^{-3} \mathrm{M}$ $[P=.02]$; on HepaRG progenitors: $25 \%$ decrease at $10^{-4} \mathrm{M}$ $[P=.02], 40 \%$ decrease at $\left.10^{-3} \mathrm{M}[P=.04]\right), A P C$ (on HuH7: $200 \%$ increase at $10^{-4} \mathrm{M}[P=.05], 55 \%$ increase at $10^{-3} \mathrm{M}$ $[P=.03]$; and on HepaRG progenitors: not significant), and HRK (on HuH7: $180 \%$ increase at $10^{-4} \mathrm{M}[P=.04], 220 \%$ increase at $10^{-3} \mathrm{M}[P<.01]$ and on HepaRG progenitors: $58 \%$ increase at $10^{-4} \mathrm{M}[P=.02], 137 \%$ increase at $10^{-3} \mathrm{M}$ $[P<.01])$ genes, not only in HuH7 cells but also in HepaRG cells treated with different concentrations of ropivacaine (Figure 5). Decreased expression CCNB1 and increased expression of HRK were more pronounced in $\mathrm{HuH7}$ cells as compared to HepaRG cells (Figure 5). Western-blot analysis confirmed a decrease in the expression of cyclin A (on HuH7: 30\% decrease at $10^{-4} \mathrm{M}[P=.05], 50 \%$ decrease at $10^{-3} \mathrm{M}[P=.04]$ and on HepaRG progenitors: $34 \%$ decrease at $10^{-3} \mathrm{M}[\mathrm{P}=.02]$ ) and cyclin $\mathrm{B}$ (on HuH7: $70 \%$ decrease at $10^{-4} \mathrm{M}[P=.01], 80 \%$ decrease at $10^{-3} \mathrm{M}[P<.01]$ and on HepaRG progenitors: $35 \%$ decrease at $10^{-4} \mathrm{M}[P=.02]$, $65 \%$ decrease at $\left.10^{-3} \mathrm{M}[P<.01]\right)$ at a protein level in both cell lines (Supplemental Digital Content 6, Figure 3, http: / / links.lww.com/AA/B973). Increased expression of proapoptotic HRK genes (Figure 5) after ropivacaine treatment correlated with an increased caspase activity and apoptosis (on HuH7: 173\% increase at $10^{-4} \mathrm{M}[P<.01], 217 \%$ increase at $10^{-3} \mathrm{M}[P<.001]$ and on HepaRG progenitors: $53 \%$ increase at $10^{-4} \mathrm{M}[P=.03], 69 \%$ increase at $10^{-3} \mathrm{M}[P<.01]$ ) (Figure 6; Supplemental Digital Content 3, Table 2, http:/ /links.lww. com/AA/B970). No effect on senescence was observed (Supplemental Digital Content 7, Figure 4, http://links. lww.com/AA/B974). Altogether, these results indicated that ropivacaine treatment resulted in inhibition of tumor cell growth by inducing cell cycle arrest and apoptosis.

\section{DISCUSSION}

To our knowledge, this study is the first report on the antitumor effect of LAs on HCC cells. LAs were previously shown to inhibit cell growth and induce cell death in lung, ${ }^{7}$ colon, ${ }^{21}$ and pancreatic cancer cells. ${ }^{22}$

In healthy tissue, cell growth and cell cycle are tightly regulated. The loss of this regulation due to gene mutation (eg, inactivation of the tumor suppressor gene TP53), epigenetic or genomic deregulation, is a hallmark of cancer cells and results in their uncontrolled proliferation, associated with apoptosis resistance. In the present study, we show that ropivacaine may stop the G2 phase of the cell cycle in HCC cells. Only a few reports have investigated the effects of LAs on the cell cycle. In noncancer cells, Lucchinetti et $\mathrm{al}^{23}$ showed that lidocaine and bupivacaine inhibit the cell cycle of mesenchymal stem cell at the G1/S phase transition. In colon and pancreatic cancer cell lines, Bundscherer et $\mathrm{al}^{22}$ observed a significant antiproliferative effect with of high concentrations of ropivacaine and bupivacaine. Ropivacaine was shown to inhibit colon cancer cells' voltage-gated sodium channels (NaV1.5) and metastatic colon cancer cell invasion. ${ }^{8}$ In our study, ropivacaine inhibited the proliferation of HCC cells by stopping the cell cycle in G2 phase. It decreased the mRNA abundance of key cell cycle regulators, especially involved in the G2-M transition phase, namely cyclin A2, cyclin B1, cyclin B2, and cyclindependent kinase 1 . Ropivacaine also decreased the expression of MKI67, a nuclear marker of cell proliferation. Indeed, the CDK1-cyclin A complex allows the cell cycle to progress from the $S$ (DNA replication) to the G2 (preparation for cell 


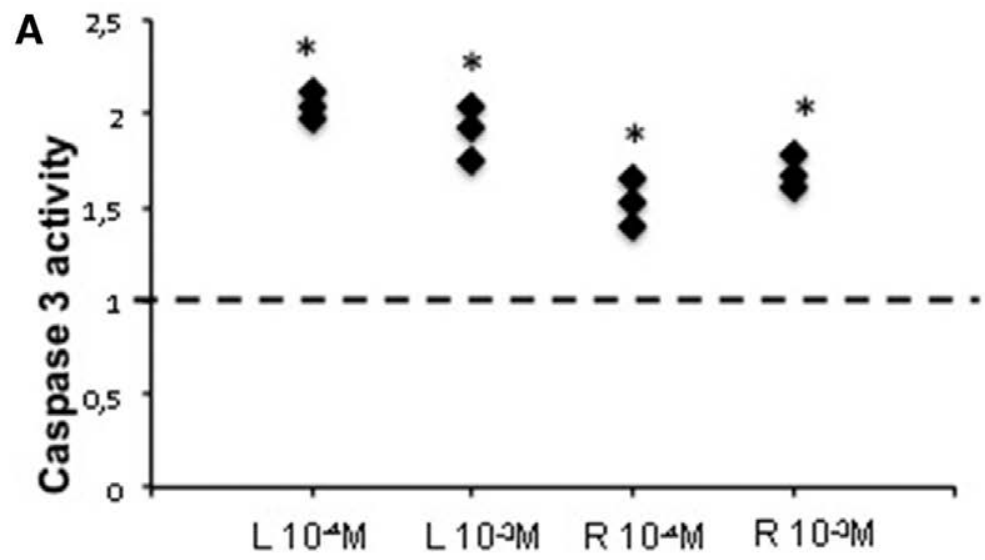

Figure 6. Effects of LAs on apoptosis of HepaRG progenitor (A) and Huh7 (B) cells. The apoptotic effect was assessed by determining caspase 3 activity. HuH7 and HepaRG progenitor cells were cultured with or without LAs for $48 \mathrm{~h}$; Caspase 3 activity was upregulated by lidocaine with a stronger effect than ropivacaine; results were confirmed when adjusted on cell viability (MTT assay). Data from 3 independent experiments shown as scatter plot (dashed line $=1$, representing control). Data are expressed as percentage of the control $* P<.05$ compared with control. $L$ indicates lidocaine; LA, local anesthetics; $R$, ropivacaine.

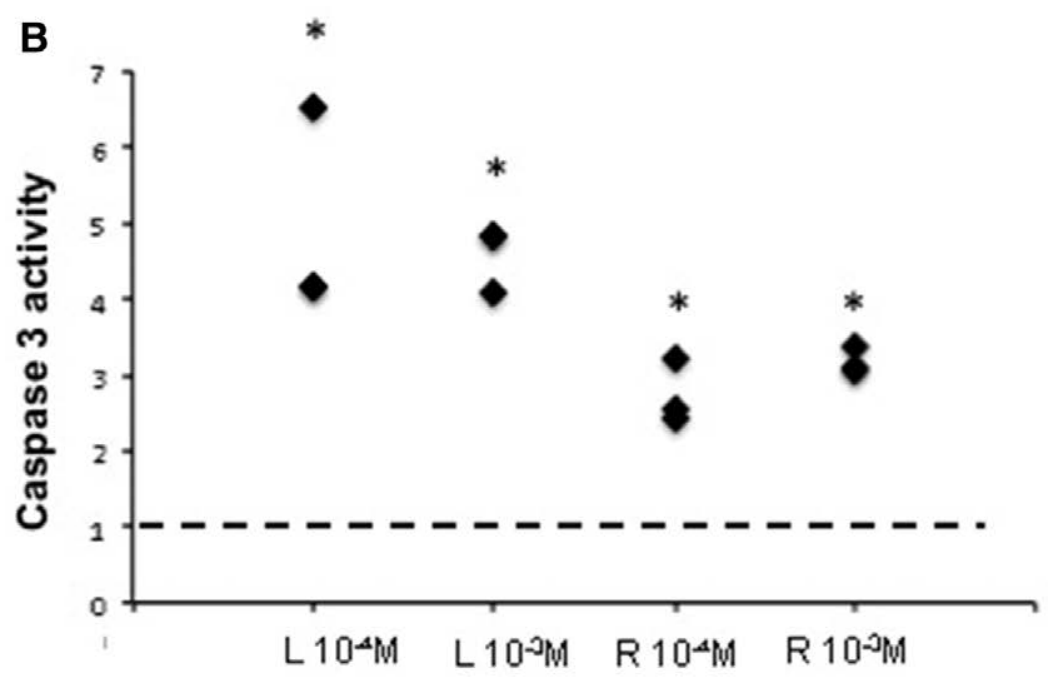

division) phase and the CDK1-cyclin B complex allows the progression from G2 to M (cell division) phase. Moreover, ropivacaine increased apoptosis in HepaRG progenitor cells without any effect on cellular senescence. In our study, the effects of ropivacaine were more pronounced on $\mathrm{HuH7}$ and HepaRG progenitor cells than on differentiated HepaRG cells. These observations suggest an enhanced effect of LA on highly versus poorly proliferated cells.

In noncancer cells, lidocaine at a high concentration has been shown to stop cell cycle at the $S$ phase and to inhibit fibroblast multiplication. ${ }^{24}$ In breast cancer cells, a potential antitumor effect of lidocaine was reported, associated with a demethylation effect ${ }^{25}$ and a sensitization effect to cisplatin cytotoxicity. ${ }^{26}$ In vitro, lidocaine enhanced natural killer cell cytotoxicity against lymphoblast cells at 0.01 and $0.1 \mu \mathrm{M} .{ }^{27}$ Recently, Chang et $\mathrm{al}^{28}$ reported that lidocaine and bupivacaine are cytotoxic for thyroid cancer cells. These 2 LAs damaged the mitochondrial membrane potential, lead to cytochrome $C$ release, activation of caspases 3 and 7, poly(ADP-ribose) polymerase cleavage, and induction of BCI-2 associated X. In our study, lidocaine increased by 10 times the mRNA levels of $A P C$ and of $D K K 1$, which both act as antagonists of the Wnt $/ \beta$-catenin pathway. The effect of lidocaine on $D K K 1$ was more modest than on $A P C$. The Wnt/ $\beta$-catenin pathway is long known to be involved in carcinogenesis, especially in HCC. ${ }^{29,30}$ Therefore, lidocaine could be of interest in HCC, particularly those subtypes with an increase activity of the Wnt/ $\beta$-catenin pathway.

In our study, the effects and the underlying mechanisms of action of lidocaine and ropivacaine were different. Different effects of each LA have been previously reported on different cell lines including T-cells, ${ }^{31}$ neuronal cells, ${ }^{32}$ and mesenchymal stromal cells. ${ }^{33}$ In lung cancer cells, ropivacaine and lidocaine inhibiting effects on Src were mediated through different pathways. ${ }^{7}$ Indeed, LAs possess distinct chemical structures and properties. Jose et $\mathrm{al}^{34}$ previously reported a cell-type- and molecule-type-specific effect. In their study, levobupivacaine triggered a more potent cancer-specific reduction of viability than ropivacaine on certain cancer cell type. They hypothesized a different effect on mitochondrial respiratory chain and ATP synthesis. Moreover, the systemic anti-inflammatory effects of bupivacaine are not mediated through sodium channel inhibition $^{35}$ indicating that LAs exert their properties by acting on a variety of targets. The differences observed in previous studies as well as in the present study regarding ropivacaine and lidocaine remain mostly unexplained on a mechanistic level and need further experiments.

The effects of LAs on cell viability, cell cycle, genes, and pathways deregulation are dose dependent. ${ }^{7}$ We observed the cytostatic effects of LAs on HCC cells for concentration ranging from $10^{-2}$ to $10^{-5} \mathrm{M}$. In vitro concentrations vary 
widely in the previous studies. Effects on cancer cells have been described for concentrations ranging from $10^{-4} \mathrm{M}^{7}$ to $13.5 \times 10^{-3} \mathrm{M}^{36}$ for lidocaine and from $10^{-5} \mathrm{M}^{22}$ to $4.32 \times 10^{-3}$ $\mathrm{M}^{37}$ for ropivacaine. High concentrations of LAs are cytotoxic for noncancer human cells. Indeed, only few studies have tested the viability of control cells when LAs are added. Chang et al $^{36}$ used mammary epithelial cells as controls and reported toxicity with higher concentrations when compared with breast cancer cells. However concentrations were high in this study.

Many mechanisms by which LAs and regional anesthesia could exert an antitumor effect have been suggested in the literature: (1) a decrease in opioid requirement is always associated with the use of LAs and opioid might promote cancer cells proliferation ${ }^{38}$; (2) regional anesthesia inhibits axonal transport ${ }^{39}$ and therefore could stop the dissemination of cancer cells during surgery; (3) a direct inhibition of cancer cells growth by LAs. ${ }^{7}$ Clinical studies on the potential benefit of LAs during cancer surgery have been published for more than 10 years. Although retrospective and with some methodological bias, these studies lead to the hypothesis that LAs could mitigate perioperative tumor growth and metastasis formation, ${ }^{2,4,5}$ specifically in the setting of breast and prostate cancer. These clinical studies are echoing the experimental studies that have already reported a reduction of tumor cell growth by LAs in the specific settings of thyroid, ${ }^{28}$ breast, ${ }^{25}$ lung, ${ }^{7}$ and colon ${ }^{8}$ cancer for example. Moreover, Lucchinetti et $\mathrm{al}^{23}$ showed how LAs impaired the proliferation of mesenchymal stem cell, which are known to play an important role in tumor progression. ${ }^{40}$ This effect was associated with a potential detrimental effect on wound healing when LAs are administered directly on the wound. Our work is the first to report a mechanism of the inhibiting effect of 2 LAs on HCC cells. In addition to potential effects in the tumor microenvironment, our results showed that LAs may induce profound modifications in gene expression profiles of tumor cells, notably by modulating cell cycle-related genes resulting in a cytostatic effect and induction of apoptosis. Multiple pathways are involved in the modulation of cell growth. These pathways cross talk to modulate the balance between proliferation and apoptosis. Based on the literature, it is difficult to determine if there is a waterfall effect induced by LAs on these pathways. ${ }^{23}$ However, the antiproliferative effect of LAs on HCC cells has to be balanced with the possible risks of LAs toxicity and wound healing impairment. Moreover, our results would transpose with difficulty in the clinical setting. Indeed, many elements such as the absence of stress and/or inflammation and/or opioids and/or pain could eventually interact with the effect of LAs. Both preclinical and clinical studies are required to further confirm the benefit of LAs on the outcome of HCC surgery. Due to its analgesic properties, intravenous lidocaine is already part of most anesthesia protocol for abdominal surgery. Studying its effect on preventing cancer recurrence would therefore be feasible in clinical practice.

\section{ACKNOWLEDGMENTS}

The authors thank Rémy le Guével from the "ImPACcell" platform (Biogenouest, SFR biosit, University of Rennes1) and Isabelle Cannie for technical assistance.

\section{DISCLOSURES}

Name: Grégoire Le Gac, MD.

Contribution: This author helped conduct the study.

Name: Gaëlle Angenard, BS.

Contribution: This author helped conduct the study.

Name: Bruno Clément, $\mathrm{PhD}$.

Contribution: This author helped analyze the data and write the manuscript.

Name: Bruno Laviolle, MD, PhD.

Contribution: This author helped analyze the data and correct the statistics.

Name: Cédric Coulouarn, PhD.

Contribution: This author helped conduct the study, analyze the data, and write the manuscript.

Name: Hélène Beloeil, MD, PhD.

Contribution: This author helped conduct the study, analyze the data, and write the manuscript.

This manuscript was handled by: Scott M. Fishman, MD.

\section{REFERENCES}

1. Gottschalk A, Sharma S, Ford J, Durieux ME, Tiouririne M. Review article: the role of the perioperative period in recurrence after cancer surgery. Anesth Analg. 2010;110:1636-1643.

2. Biki B, Mascha E, Moriarty DC, et al. Anesthetic technique for radical prostatectomy surgery affects cancer recurrence: a retrospective analysis. Anesthesiology. 2008;109:180-187.

3. Vigneault L, Turgeon AF, Côté D, et al. Perioperative intravenous lidocaine infusion for postoperative pain control: a meta-analysis of randomized controlled trials. Can J Anaesth. 2011;58:22-37.

4. Exadaktylos AK, Buggy DJ, Moriarty DC, Mascha E, Sessler DI. Can anesthetic technique for primary breast cancer surgery affect recurrence or metastasis? Anesthesiology. 2006;105:660-664.

5. Cummings KC III, Xu F, Cummings LC, Cooper GS. A comparison of epidural analgesia and traditional pain management effects on survival and cancer recurrence after colectomy: a population-based study. Anesthesiology. 2012;116:797-806.

6. Chen WK, Miao CH. The effect of anesthetic technique on survival in human cancers: a meta-analysis of retrospective and prospective studies. PLoS One. 2013;8:e56540.

7. Piegeler T, Votta-Velis EG, Liu G, et al. Antimetastatic potential of amide-linked local anesthetics: inhibition of lung adenocarcinoma cell migration and inflammatory Src signaling independent of sodium channel blockade. Anesthesiology. 2012;117:548-559.

8. Baptista-Hon DT, Robertson FM, Robertson GB, et al. Potent inhibition by ropivacaine of metastatic colon cancer SW620 cell invasion and NaV1.5 channel function. $\mathrm{Br} J$ Anaesth. 2014;113(suppl 1):i39-i48.

9. Tada M, Imazeki F, Fukai K, et al. Procaine inhibits the proliferation and DNA methylation in human hepatoma cells. Hepatol Int. 2007;1:355-364.

10. European Association for Study of Liver; European Organisation for Research and Treatment of Cancer. EASL-EORTC clinical practice guidelines: management of hepatocellular carcinoma. Eur I Cancer. 2012;48:599-641.

11. Llovet JM, Fuster J, Bruix J. Intention-to-treat analysis of surgical treatment for early hepatocellular carcinoma: resection versus transplantation. Hepatology. 1999;30:1434-1440.

12. Nakabayashi H, Taketa K, Miyano K, Yamane T, Sato J. Growth of human hepatoma cells lines with differentiated functions in chemically defined medium. Cancer Res. 1982;42:3858-3863.

13. Cerec V, Glaise D, Garnier D, et al. Transdifferentiation of hepatocyte-like cells from the human hepatoma HepaRG cell line through bipotent progenitor. Hepatology. 2007;45:957-967.

14. Coulouarn C, Corlu A, Glaise D, et al. Hepatocyte-stellate cell cross-talk in the liver engenders a permissive inflammatory microenvironment that drives progression in hepatocellular carcinoma. Cancer Res. 2012;72:2533-2542.

15. Dubois-Pot-Schneider H, Fekir K, Coulouarn C, et al. Inflammatory cytokines promote the retrodifferentiation of tumor-derived hepatocyte-like cells to progenitor cells. Hepatology. 2014;60:2077-2090. 
16. Gripon P, Rumin S, Urban $S$, et al. Infection of a human hepatoma cell line by hepatitis B virus. Proc Natl Acad Sci U S A. 2002;99:15655-15660.

17. Denizot F, Lang R. Rapid colorimetric assay for cell growth and survival. Modifications to the tetrazolium dye procedure giving improved sensitivity and reliability. J Immunol Methods. 1986;89:271-277.

18. Schneider-Jakob S, Corazza N, Badmann A, et al. Synergistic induction of cell death in liver tumor cells by TRAIL and chemotherapeutic drugs via the BH3-only proteins Bim and Bid. Cell Death Dis. 2010;1:e86.

19. Sulpice L, Rayar M, Desille M, et al. Molecular profiling of stroma identifies osteopontin as an independent predictor of poor prognosis in intrahepatic cholangiocarcinoma. Hepatology. 2013;58:1992-2000.

20. Wright GW, Simon RM. A random variance model for detection of differential gene expression in small microarray experiments. Bioinformatics. 2003;19:2448-2455.

21. Martinsson T. Ropivacaine inhibits serum-induced proliferation of colon adenocarcinoma cells in vitro. J Pharmacol Exp Ther. 1999;288:660-664.

22. Bundscherer A, Malsy M, Gebhardt K, et al. Effects of ropivacaine, bupivacaine and sufentanil in colon and pancreatic cancer cells in vitro. Pharmacol Res. 2015;95-96:126-131.

23. Lucchinetti E, Awad AE, Rahman M, et al. Antiproliferative effects of local anesthetics on mesenchymal stem cells: potential implications for tumor spreading and wound healing. Anesthesiology. 2012;116:841-856.

24. Desai SP, Kojima K, Vacanti CA, Kodama S. Lidocaine inhibits NIH-3T3 cell multiplication by increasing the expression of cyclin-dependent kinase inhibitor 1A (p21). Anesth Analg. 2008;107:1592-1597.

25. Lirk P, Hollmann MW, Fleischer $M$, Weber NC, Fiegl $H$ Lidocaine and ropivacaine, but not bupivacaine, demethylate deoxyribonucleic acid in breast cancer cells in vitro. $\mathrm{Br}$ Anaesth. 2014;113(suppl 1):i32-i38.

26. Li K, Yang J, Han X. Lidocaine sensitizes the cytotoxicity of cisplatin in breast cancer cells via up-regulation of RAR $\beta 2$ and RASSF1A demethylation. Int J Mol Sci. 2014;15:2351923536.
27. Ramirez MF, Tran P, Cata JP. The effect of clinically therapeutic plasma concentrations of lidocaine on natural killer cell cytotoxicity. Reg Anesth Pain Med. 2015;40:43-48.

28. Chang YC, Hsu YC, Liu CL, et al. Local anesthetics induce apoptosis in human thyroid cancer cells through the mitogenactivated protein kinase pathway. PLoS One. 2014;9:e89563.

29. Clevers H, Nusse R. Wnt/ $\beta$-catenin signaling and disease. Cell. 2012;149:1192-1205.

30. Monga SP. $\beta$-catenin signaling and roles in liver homeostasis, injury, and tumorigenesis. Gastroenterology. 2015;148:1294-1310.

31. Boselli E, Duflo F, Debon R, et al. The induction of apoptosis by local anesthetics: a comparison between lidocaine and ropivacaine. Anesth Analg. 2003;96:755-756.

32. Perez-Castro R, Patel S, Garavito-Aguilar ZV, et al. Cytotoxicity of local anesthetics in human neuronal cells. Anesth Analg. 2009;108:997-1007.

33. Gray A, Marrero-Berrios I, Ghodbane M, et al. Effect of local anesthetics on human mesenchymal stromal cell secretion. Nano Life. 2015;5:1550001-1550014.

34. Jose C, Bellance N, Chatelain EH, et al. Antiproliferative activity of levobupivacaine and aminoimidazole carboxamide ribonucleotide on human cancer cells of variable bioenergetic profile. Mitochondrion. 2012;12:100-109.

35. Beloeil H, Ababneh Z, Chung R, et al. Effects of bupivacaine and tetrodotoxin on carrageenan-induced hind paw inflammation in rats (part 1): hyperalgesia, edema, and systemic cytokines. Anesthesiology. 2006;105:128-138.

36. Chang YC, Liu CL, Chen MJ, et al. Local anesthetics induce apoptosis in human breast tumor cells. Anesth Analg. 2014;118:116-124.

37. Werdehausen R, Fazeli S, Braun S, et al. Apoptosis induction by different local anaesthetics in a neuroblastoma cell line. $\mathrm{Br} J$ Anaesth. 2009;103:711-718.

38. Afsharimani B, Cabot P, Parat MO. Morphine and tumor growth and metastasis. Cancer Metastasis Rev. 2011;30:225-238.

39. Deruddre S, Combettes E, Estebe JP, et al. Effects of a bupivacaine nerve block on the axonal transport of tumor necrosis factor-alpha (TNF-alpha) in a rat model of carrageenan-induced inflammation. Brain Behav Immun. 2010;24:652-659.

40. Wong RS. Mesenchymal stem cells: angels or demons? J Biomed Biotechnol. 2011;2011:459510. 